\title{
Error Model-converted Measurement and Error Model-modified Extended Kalman Filters for Target Tracking
}

\author{
Sudesh K. Kashyap, G. Cirija and J.R. Raol \\ National Aerospace Laboratories, Bangalore-560 017
}

\begin{abstract}
Two-filter schemes have been evaluated to handle the polar measurements using error model (for bias correction and measurement noise covariance computation) for target-tracking application. It is assumed that a good reference source of target information is available. Schemes based on error model converted measurement Kalman filter (ECMKF) and error model modified extended-Kalman filter (EMEKF) algorithms are presented. Also some comparison with CMKF (debiased) is given. It is inferred that EMEKF gives better performance compared to other filters. Features of CMKF (debiased), ECMKF, and EMEKF are highlighted. Also the sensitivity study on the performance of EMEKF is carried out wrt to processing order of radar measurement channels.
\end{abstract}

Keywords: Polar measurements, target tracking, radar measurements, filter scheme, ECMKF, CMKF, EMEKF, algorithms, Kalman filter, target algorithms, Cartesian coordinate frames,polar frame

\section{INTRODUCTION}

Algorithms for obtaining improved accuracy with radar measurements in target-tracking applications using Kalman filters are available in the literature". Target dynamics are best described in Cartesian coordinate frame, since the dynamic equations are uncoupled and lincar. However, radars measure the target range, elevation and azimuth, resulting in a nonlinear relation between the states and the measurements. It is known that, the inaccuracies of the measurements have a direct effect on the performance of the tracking algorithms. For target tracking using the radar measurements, two approaches are commonly used. The first approach is a linear Kalman filter (CMKF), wherein the measurements used for updating the states are generated by converting the raw measurements in polar frame to the Cartesian frame so that the measurements are linear functions of the states? In this case, the converted measurement errors get correlated. Also, when the cross-range measurement errors are large, the mean of the errors is high, and hence, debiasing is required. The measurement noise covariance matrix should include cross-covariance terms to account for correlated measurement errors. Analytical expressions for debiased consistent estimates (CMKF-D) have been derived ${ }^{2}$. Implementation necessitates the evaluation of complex equations. The second approach is an extended-Kalman filter (EKF), wherein the measurements used for updating the states arc the range, azimuth, and elevation in polar frame so that one has a filter where the measurements are nonlinear functions of the states resulting in a mixed coordinate filter. It is well-known that in 
the EKF, the initial covariance depends on the initial converted measurements and the gains depend on the accuracy of the subsequent linearisation. The overall performance depends on these accuracies. A simple way to handle the nonlinearities has been proposed?. The method involves sequential processing of the radar measurements in elevation, azimuth, and range while linearising the nonlinear equations wrt the estimated states at each instant of time. The sequential processing results in considerable computational savings. However, when the nonlinearities are significant, modified expressions for the mean and covariance errors are used and a modified (measurements arc sequentially processed) EKF (called MEKF) was proposed'. Thus, for achieving better accuracies, both the methods require certain modifications to handle bias as well as mcasurement error covariances in the conventional linear Kalman filter as well as in EKF.

An alternative way of achieving dcbiasing and obtaining an estimate of the measurement error covariances using converted radar measurements is proposed. This method, termed ECMKF (error model converted measurement Kalman filter) presupposes the availability of a very accurate reference data from an independent measurement source. It is known that GPS gives very accurate measurements of position of, eg, aircraft. Hence, it can be used to get accurate estimates of the bias as well as measurement noise covariance of the converted measurcments in the Cartesian frame using Kalman filter with error state space formulation ${ }^{5}$. The estimated bias is used to correct the converted mcasurements. and the estimated covariance values are used in the ECMKF.

The performance of the ECMKF is compared with the EMEKF algorithm which handles measurements of range, azimuth, and elevation directly using simulated data ofa target with different measurement accuracies. The algorithms are implemented in PC MATLAB. The algorithms are evaluated for their performances in terms of root sum square position error (RSSPE), and fit error wrt true data. The algorithms are also used for tracking a moving aircraft from the ground-based radar measurements when GPS measurements of position of the aircraft arc available. For the sake of comparison, the results of $\mathrm{CMKF}-\mathrm{D}^{2}$ are also presented $^{6}$.

\section{ECMKF AND EMEKF ALGORITHMS}

'Target motion model is described in the Cartesian coordinate system by linear discrete-time difference equation with additive noise as

$$
X_{k+1}=F X_{k} \mathrm{t} G w_{k}
$$

where the Cartesian state vector $(X)$ consists of the position and velocity of the target moving in 3-D space, ie, $X=\left[\begin{array}{llllll}x & y & z & \times & y & \dot{z}\end{array}\right]$ and the process noise $(w)$ is assumed to be white and zero mean with covariance $(Q)$. The target is tracked by a ground-based radar and provides measurements of range $\left(r_{m}\right)$, azimuth $\left(\theta_{m}\right)$ and elevation $\left(\phi_{m}\right)$. The measurement model is given as

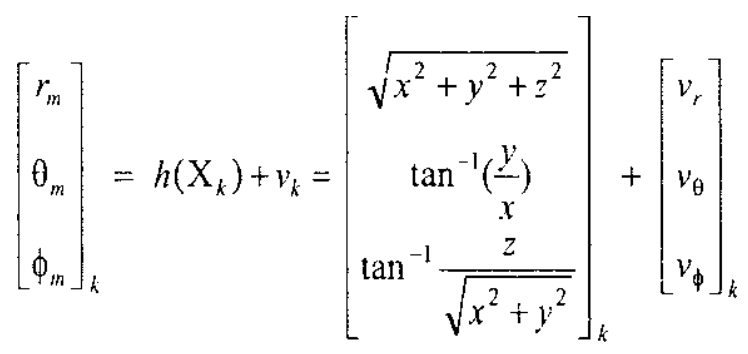

where $v_{i}, v_{\theta}, v_{\phi}$ are assumed to be mutually uncorrelated and zero-mean white Gaussian noise with variances $\sigma_{r}^{2}, \sigma_{\theta}^{2}, \sigma_{\phi}^{2}$, respectively.

\subsection{ECMKF Algorithm}

The measured range, azimuth, and clcvation from radar are converted to positions in Cartesian frame using the following relations:

$$
\begin{aligned}
& x=r_{m} * \cos \left(\phi_{m}\right) * \cos \left(\theta_{m}\right) \\
& y=r_{m} * \cos \left(\phi_{m}\right) * \sin \left(\theta_{m}\right) \\
& z=r_{m} * \sin (\$,,) \\
& \mathrm{Z},,=\left[\begin{array}{lll}
x & y & z
\end{array}\right]
\end{aligned}
$$

\section{Error Model Kalman Filter}

With the GPS data as reference and the converted measurement data, the objective is to utilise the information and combine the same in an optimal 


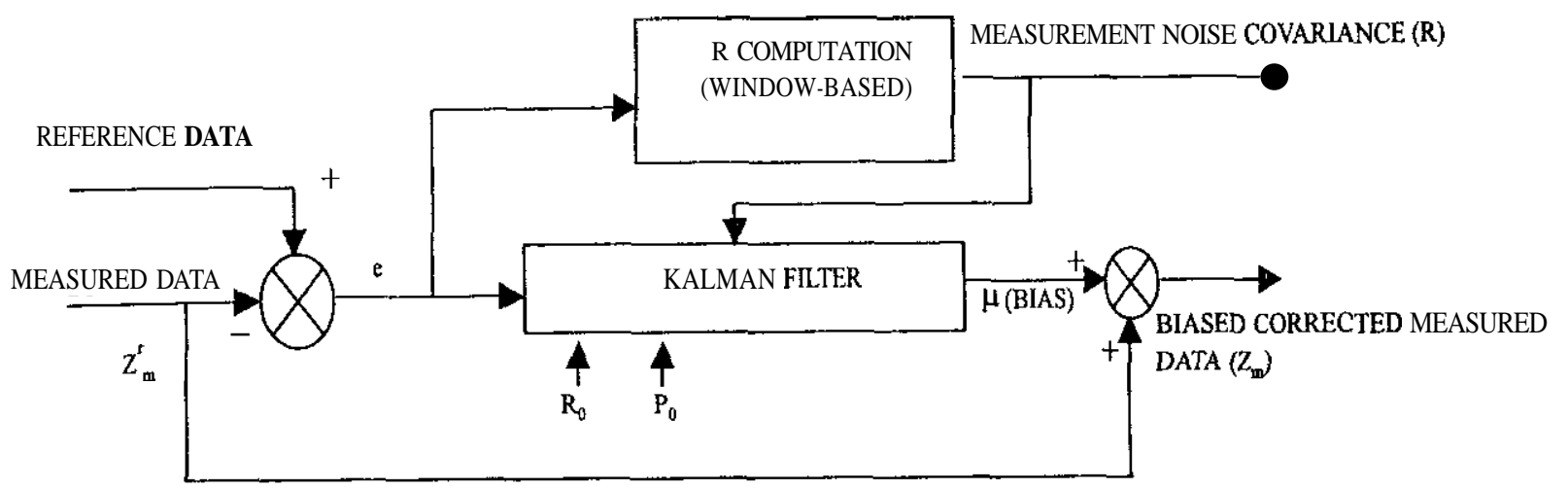

Figure 1(a). Error model Kalman filler.

manner to estimate the bias errors in the sensor measurements and obtain estimate of the measurement noise covariance for the sensors. Although GPS would be accurate, there would be some outages due to atmosphere disturbances/obstacles in the path of the signal. Hence GPS signal only cannot be used for target tracking. Also GPS signal is available at one second interval and radar data are available at much faster rate, and hence, for accurate results, $\mathrm{KF}$ is needed to process radar data. Kalman filter for this purpose has the advantage of using the statistical characteristics of the errors in both the reference data and other sensors to determine the optimal estimate of the bias characteristics. In this application, the Kalman filter uses error state space formulation in place of the actual state space forinulation [Fig.I(a)]. The error state space Kalman filter estimates the errors in the converted sensor data using the difference between the measured position data and the reference data (supposed to be accurate and independent source data). Error model Kalman filter (EMKF) gives optimal estimates of the errors $(\delta \hat{X})$ in the sensors-based on the error state model it carries, the difference between the converted radar data and the GPS reference data and the noise statistics. The error estimates are used to correct the measurement data. The error state model in discrete form is given as'

$$
\begin{aligned}
& \delta X_{K+1}=F \delta X_{K}+G w_{K} \\
& \delta Z_{K}=H \delta X_{K}+v_{K}
\end{aligned}
$$

where $\delta X$ is the vector of position and velocity crrors in all the three axes, $\delta Z$ is the vector of computed position error in all the three axes (GPSradar measurement), $F$ is the transition matrix, $H$ is the output matrix, $w$ is the process noise with mean zero and variance $\mathrm{Q}$, and $\boldsymbol{v}$ is the measurement noise with mean zero and variance $\mathrm{R}$. The estimated position error is used to correct the converted data which is used for the measurement update. Tine ECMKF equations follow the conventional lincar Kalman filter.

\section{Initialisation}

$$
X_{010}=X_{0} \text {, }
$$

Time Propagation

$$
\begin{aligned}
& \tilde{X}_{k \mid k-1}=F_{k-1} \hat{X}_{k-1 \mid k-1} \\
& \tilde{P}_{k \mid k-1}=F_{k-1} \hat{P}_{k-1 \mid k-1} F_{k-1}^{T}+G_{k-1} Q_{k-1} G_{k-1}^{T}
\end{aligned}
$$

Measurement Updaie

$$
\begin{aligned}
& S=H \widetilde{P}_{k \mid k-1} H^{T}+R \\
& K=\widetilde{P}_{k \mid k-1} H^{T}(S)^{-1} \\
& \hat{X}_{k \mid k}=\widetilde{X}_{k \mid k \cdot 1}+K\left(Z_{m}-H \widetilde{X}_{k \mid k-1}\right) \\
& \hat{P}_{k \mid k}=\widetilde{P}_{k \mid k-1}-K S K^{T}
\end{aligned}
$$

Error statc/states used in above equations are commonly used for error model/KF. The scheme is shown in block diagram of Fig. I (b) for ECMKF.

\subsection{EMEKF Algorithm-Measurement Data Updates}

In EMEKF, 3-D radar measurement vector is processed one component at a time in the preferred order of elevation, azimuth, and range, while linearising 


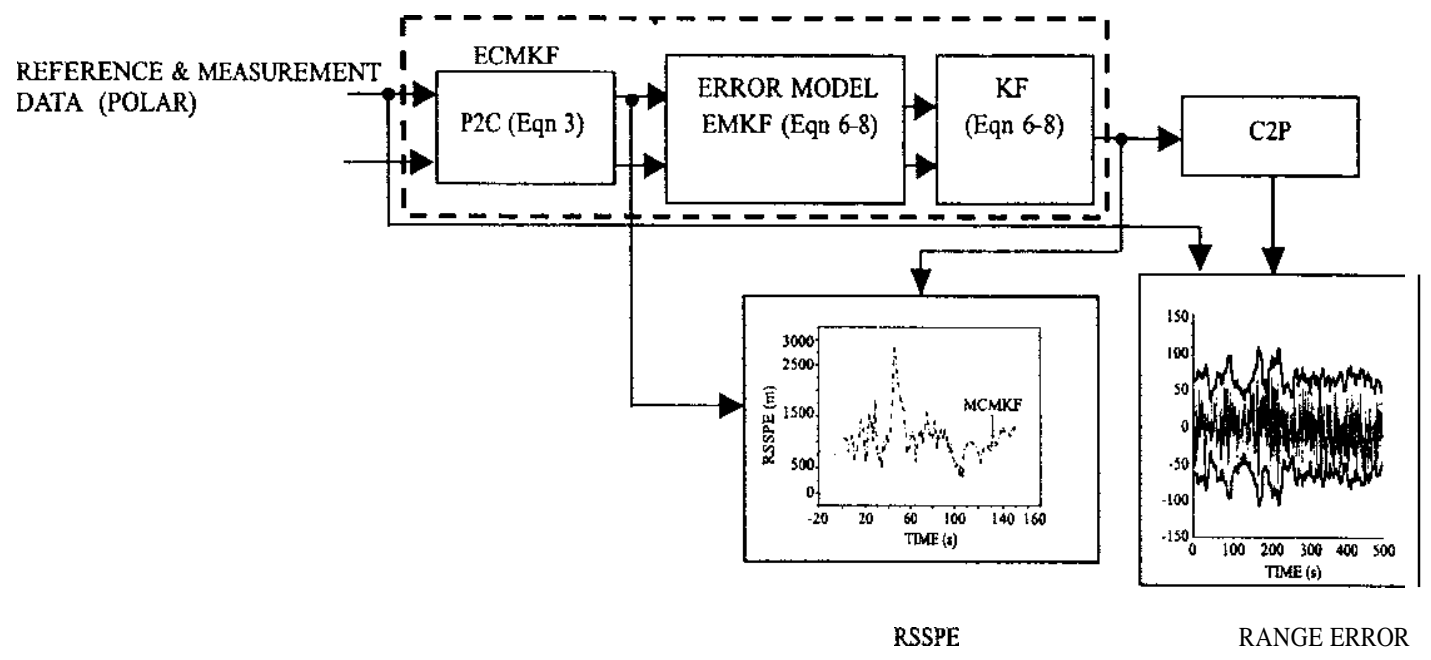

Figure 1(b). Schematic of error model converted measurement Kalman filter.

the corresponding nonlinear measurement equation wrt to the latest position estimate before each update. The initialisation and time propagation are done using Eqns (6) and (7). The error (model) states will be $\boldsymbol{r}, 0$ and $\phi$ here. It is to be noted that the measurement update the range measurement, includes extra terms in the measurement covariance part to account for nonlinear cross-coupling between the range, azimuth and elevation measurements. The following description assumes that the measurements arc processed starting with $\phi$ and predicted states, and then 0 and range'.

Update by Measurement of Elevation Data

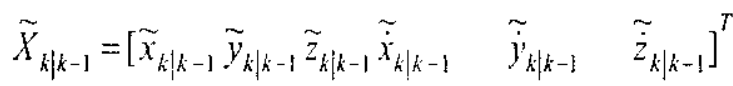

$$
\begin{aligned}
& \bar{F}_{k}=\sqrt{\left(\tilde{x}_{k \mid k-1}^{2}+\tilde{y}_{k \mid k-1}^{2}+\tilde{z}_{k \mid k-1}^{2}\right)} \\
& \bar{\theta}_{k}=\tan ^{-1}\left(\tilde{y}_{k \mid k-1} / \tilde{x}_{k \mid k-1}\right) \\
& \bar{\phi}_{k}=\tan ^{-1}\left\{\tilde{z}_{k \mid k-1} / \sqrt{\tilde{x}_{k \mid k-1}^{2}+\tilde{y}_{k \mid k-1}^{2}}\right\}
\end{aligned}
$$

$H_{k, 1}=\left[\frac{-\cos \bar{\theta}_{k} \sin \bar{\phi}_{k}}{\bar{r}_{k}} \frac{-\sin \bar{\theta}_{k} \sin \bar{\phi}_{k}}{\bar{r}_{k}} \frac{\cos \bar{\theta}_{k}}{\bar{r}_{k}} \quad 0 \quad 000\right]$ $S_{k, 1}=H_{k, 1} \widetilde{P}_{k \mid k-1} H_{k, 1}^{r}+\sigma_{\phi}^{2}$

$K_{k, 1}=\widetilde{P}_{k \mid k-1} H_{k, 1}^{T} / S_{k, 1}$

$\hat{X}_{k, 1}=\tilde{X}_{k \mid k-1}+K_{k, 1}\left(\phi_{k}^{m}-\bar{\phi}_{k}\right)$

$\hat{P}_{k, 1}=\widetilde{P}_{k \mid k-1}-K_{k, 1} S_{k, l} K_{k, 1}^{T}$
Update by Measuremeni of Azimuth Data

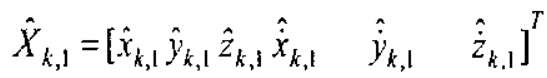

$$
\begin{aligned}
\bar{r}_{k} & =\sqrt{\left(\hat{x}_{k, 1}^{2}+\hat{y}_{k, 1}^{2}+\hat{z}_{k, 1}^{2}\right)} \\
\bar{\theta}_{k} & =\tan ^{-1}\left(\hat{y}_{k, 1} / \hat{x}_{k, 1}\right) \\
\bar{\phi}_{k} & =\tan ^{-1}\left\{\hat{z}_{k, 1} / \sqrt{\hat{x}_{k, 1}^{2}+\hat{y}_{k, 1}^{2}}\right\}
\end{aligned}
$$

$$
\begin{aligned}
H_{k, 2} & =\left[\begin{array}{llllll}
\frac{-\sin \bar{\theta}_{k}}{\bar{r}_{k} \cos \bar{\phi}_{k}} \frac{\cos \bar{\theta}_{k}}{\bar{r}_{k} \cos \bar{\phi}_{k}} & 0 & 0 & 0 & 0
\end{array}\right] \\
S_{k, 2} & =H_{k, 2} \hat{P}_{k, 1} H_{k, 2}^{T}+\sigma_{\theta}^{2} \\
K_{k, 2} & =\hat{P}_{k, 1} H_{k, 2}^{T} / S_{k, 2} \\
\hat{X}_{k, 2} & =\hat{X}_{k, 1}+K_{k, 2}\left(\theta_{k}^{m}-\bar{\theta}_{k}\right) \\
\hat{P}_{k, 2} & =\hat{P}_{k, 1}-K_{k, 2} S_{k, 2} K_{k, 2}^{T}
\end{aligned}
$$

Update by Measurement of Range Data

$$
\begin{aligned}
\hat{X}_{k, 2} & =\left[\begin{array}{lll}
\hat{x}_{k, 2} \hat{y}_{k, 2} \hat{z}_{k, 2} \hat{\dot{x}}_{k, 2} & \hat{\dot{y}}_{k, 2} & \hat{\dot{z}}_{k, 2}
\end{array}\right]^{T} \\
\bar{r}_{k} & =\sqrt{\left(\hat{x}_{k, 2}^{2}+\hat{y}_{k, 2}^{2}+\hat{z}_{k, 2}^{2}\right)} \\
\bar{\theta}_{k} & =\tan ^{-1}\left(\hat{y}_{k, 2} / \hat{x}_{k, 2}\right) \\
\bar{\phi}_{k} & =\tan ^{-1}\left\{\hat{z}_{k, 2} / \sqrt{\left.\hat{x}_{k, 2}^{2}+\hat{y}_{k, 2}^{2}\right\}}\right.
\end{aligned}
$$




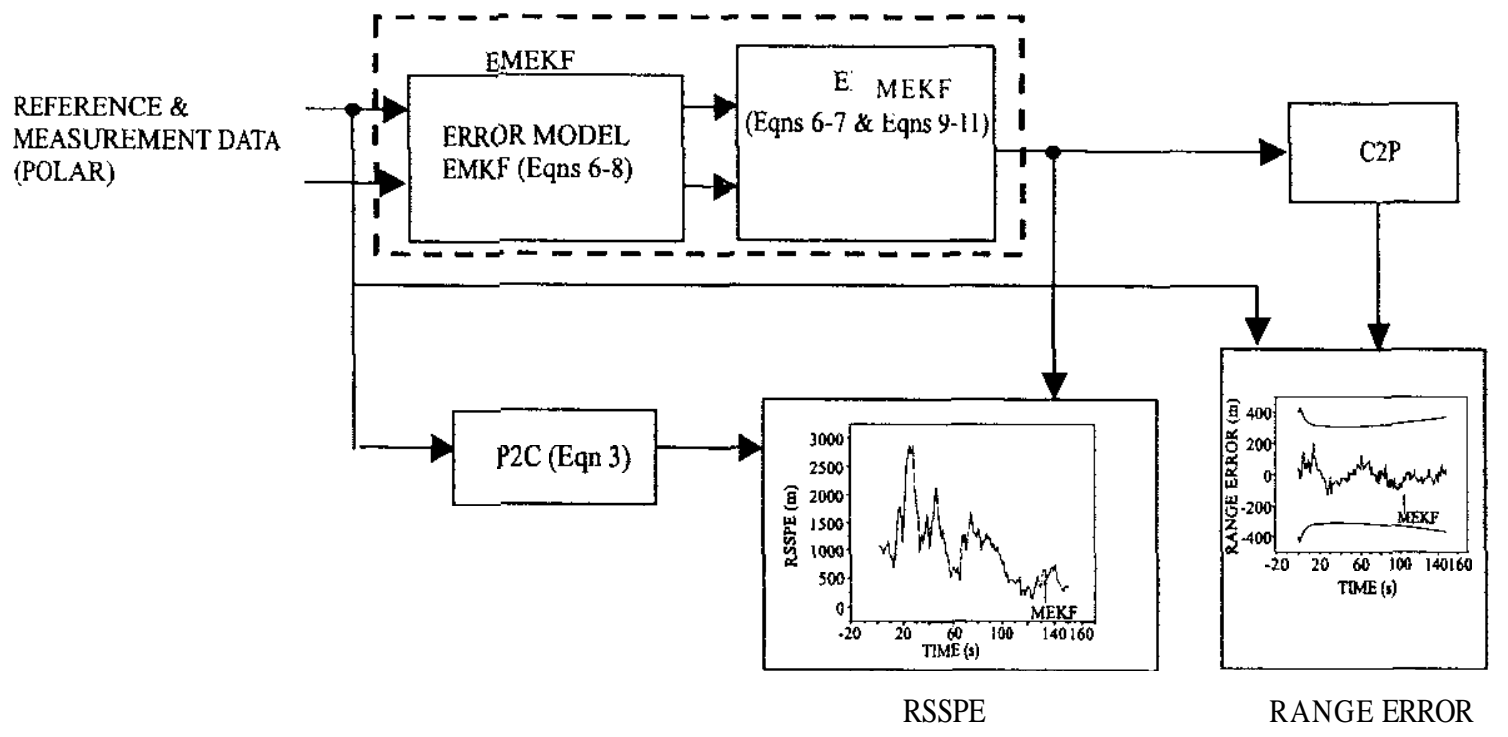

Figure 2. Schematic of error model modified extended Kalman filter: P2C is the polar to Cartesian coordinate conversion, C2P is the Cartesian to polar coordinate conversion and EMKF computes measurement bias and noise covariance.

$H_{k, 3}=\left[\begin{array}{llllll}\cos \bar{\theta}_{k} \cos \bar{\phi}_{k} & \sin \bar{\theta}_{k} \cos \bar{\phi}_{k} \sin \bar{\phi}_{k} \quad 0 \quad 0 \quad 0\end{array}\right]$

$S_{k, 3}=H_{k, 3} \hat{P}_{k, 2} H_{k, 3}^{T}+\sigma_{r}^{2}+\left(\bar{r}_{k}^{2}\left(\sigma_{\phi}^{4}+\sigma_{\theta}^{4}\right) / 2\right)$

$K_{k, 3}=\hat{P}_{k, 2} H_{k, 3}^{T} / S_{k, 3}$

$\mu_{k}^{r}=\bar{r}_{k}\left[\left(\theta_{k}^{m}-\bar{\theta}_{k}\right)^{2}+\left(\phi_{k}^{m}-\bar{\phi}_{k}\right)^{2}-\sigma_{\theta}^{2}-\sigma_{\phi}^{2}\right] / 2$

$\hat{X}_{k \mid k}=\hat{X}_{k, 2}+K_{k, 3}\left(r_{k}^{m}-\bar{r}_{k} \mu_{k}^{r}\right)$

$\hat{P}_{k \mid k}=\hat{P}_{k, 2}-K_{k, 3} S_{k, 3} K_{k, 3}^{T}$

Here $r_{k}^{m}, \theta_{k}^{m}, \phi_{k}^{m}$ are the radar measurements at $k^{\text {th }}$ scan. Figure 2 shows the block diagram of the EMEKF. For clarity and completeness, the details of CMKF-D' are also given in Appendix 1. Table I gives the features/differences in the CMKF-D,

\section{RESULT AND DISCUSSION}

The simulated data (Set 1 and Set 2) with different measurement accuracies are generated for validation purpose. The appropriate models for simulation are given as

$$
F=\left[\begin{array}{llllll}
1 & 0 & 0 & T & 0 & 0 \\
0 & 1 & 0 & 0 & T & 0 \\
0 & 0 & 1 & 0 & 0 & T \\
0 & 0 & 0 & 1 & 0 & 0 \\
0 & 0 & 0 & 0 & 1 & 0 \\
0 & 0 & 0 & 0 & 0 & 1
\end{array}\right]
$$




$\left.\begin{array}{cccccc}\mathrm{I}_{T^{2} / 2} & 0 & 0 & 0 & 0 & 0 \\ 0 & T^{2} / 2 & 0 & 0 & 0 & 0 \\ 0 & 0 & T^{2} / 2 & 0 & 0 & 0 \\ 0 & 0 & 0 & T & 0 & 0 \\ 0 & 0 & 0 & 0 & T & 0 \\ 0 & 0 & 0 & 0 & 0 & T\end{array}\right]$

$$
H=\left[\begin{array}{llllll}
1 & 0 & 0 & 0 & 0 & 0 \\
0 & 1 & 0 & 0 & 0 & 0 \\
0 & 0 & 1 & 0 & 0 & 0
\end{array}\right]
$$

where, $F, G, H$ are the state transition, process noise gain, observation matrices, respectively and $T$ is the sampling time interval in seconds.

The data is generated with the initial conditions: $\left[\begin{array}{lll}100 & -100 & 100\end{array}\right](\mathrm{m})$ for position and $\left[\begin{array}{lll}5 & -5 & 5\end{array}\right](\mathrm{m} / \mathrm{s})$ for velocity, $Q=0.25$ and 500 data points with a sampling interval of $1.0 \mathrm{~s}$ are used. Random noise is added to the true data with following standard deviations:

\section{Set 1 Data}

$$
\sigma_{r}=30 \mathrm{~m} ; \sigma_{0}=0.015^{\prime \prime} ; \sigma_{4}=0.015^{\prime \prime}
$$

\section{Set 2 Data}

$$
\sigma_{r}=30 \mathrm{~m} ; \sigma_{0}=1.5^{\circ} ; 5_{\phi}=1.5^{\prime \prime}
$$

Figures 3(a) to 3(c) (Set 1 data) and Figs 4(a) to 4(c) (Set 2 data) show the range, azimuth, and elevation errors for ECMKF and EMEKF. It is clear that the range errors are well within the theoretical bounds. Here; the bounds vary because the computation is based on windowing method. However the azimuth and elevation errors using ECMKF are outside the theoretical bounds during the initial portion of the data. Figures $3(d)$ and 4 (d) show the root sum square position error. The performance of the two algorithms shows comparable root sum square position error for Set 1 data and EMEKF indicates lower root sum square position error than the ECMKF for Set 2 data. Figures clearly show that when the angular accuracies of the measuring radar arc low, the EMEKF performs better than the ECMKF, whereas the performance of the two algorithms is roughly similar when the angular radar measurements are accurate (Tables 2 and 3). A Monte-Carlo simulation of 25 runs is also carried out on Set 2 data. The seed number for the process noise was kept constant, however, it was varied for the measurement noise, for 25 runs. The results of this exercise are given in Table 3 from which it was observed that also the average EMEKF shows better performance over the ECMKF algorithm. This is further confirmed from Figs 4(e) and 4(f).

Figures 5(a) to 5(c) show the performance of the EMEKF and ECMKF algorithms for Set 3 data [independently generated by another agency] of a moving aircraft tracked by a ground-based radar and for which accurate GPS position measurements are available. It is clear that the errors are well within the theoretical hounds. However, the EMEKF gives somewhat better performance in terms of root sum square position crror.

The performance of the ECMKF and EMEKF is evaluated in terms of percentage fit error in polar and Cartesian frames (Tables 2-4) for data (Sets 1,2 and 3), respectively. It is clear that when the angular accuracies of the measuring radar are low, the EMEKF performs better than the ECMKF.

\subsection{Sensitivity Study on EMEKF}

A 3-D radar measurement vector is processed. one component at a time, in the preferred order (based on the assumption that radar will give more accurate angular data than the range) of elevation, azimuth, and range. The effect of changing the order of measurement processing on the performance has becn studied for three cases: Casel - Elevation, azimuth, and range, Case 2 - Azimuth, range, and elevation, Case 3 - Range, elevation and azimuth. Table 5 gives statistics of the EMEKF performance for Set 2 data. The Set 2 data is used to highlight the performance of the EMEKF even when the measurement data arc highly noisy. It is clear that the measurement sequencing has little effect on the performance. 


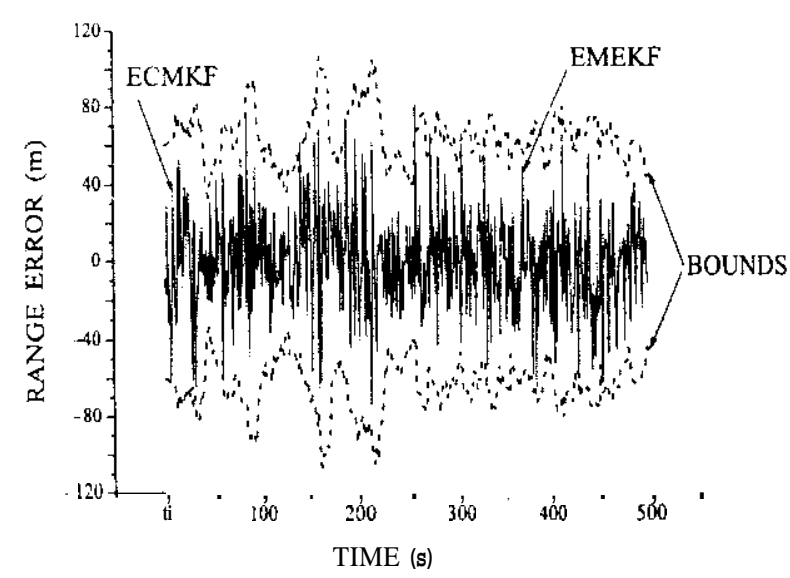

Figure 3(a). Range error comparison for EMEKF and ECMKF - simulated Set I data.

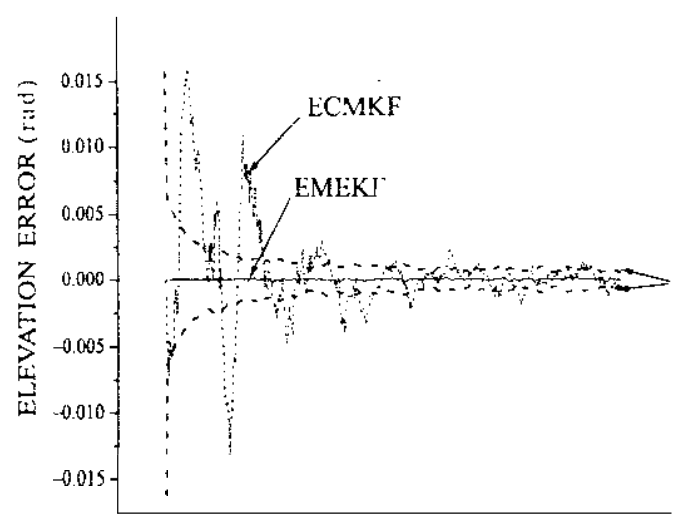

\subsection{Comparison of CMKF-D, ECMKF AND EMEKF}

For (Set 4) data, the following values arc used:

$\sigma,-30 \mathrm{~m} ; \sigma_{i}=1.5^{\circ}$

$X(0)=[100-1005-51 ; Q=0.25 ; N=500 ; t=1.0$

The results of Set 4 data processed in the CMKF-D (Appendix 1) are shown in Table 6 which also compares the performance of other algorithms. Figure 6 shows comparison of the estimated measurement noise covariance using the three techniques. The results show that

- ECMKF has better performance as compared to CMKF-D-T/CMKF-D-M in terms ofpercentage fit error.

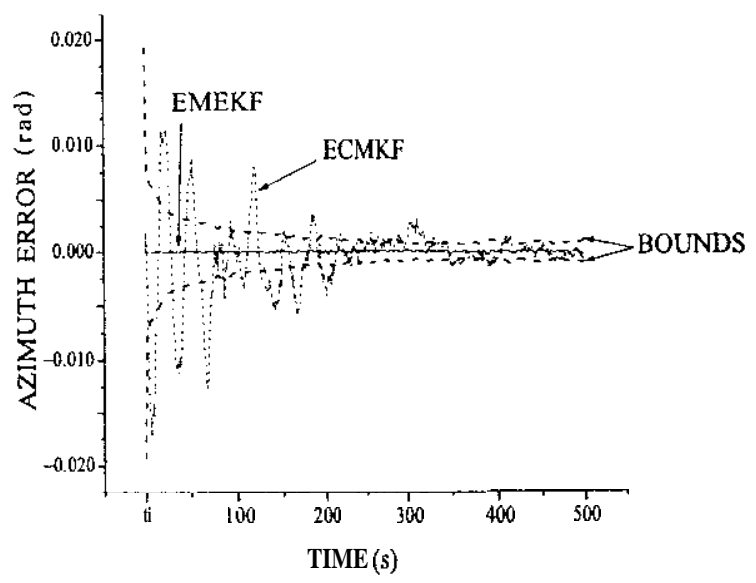

Figure 3(b). Azimuth error comparison for EMEKF and ECMKF - simulated Set 1 data.

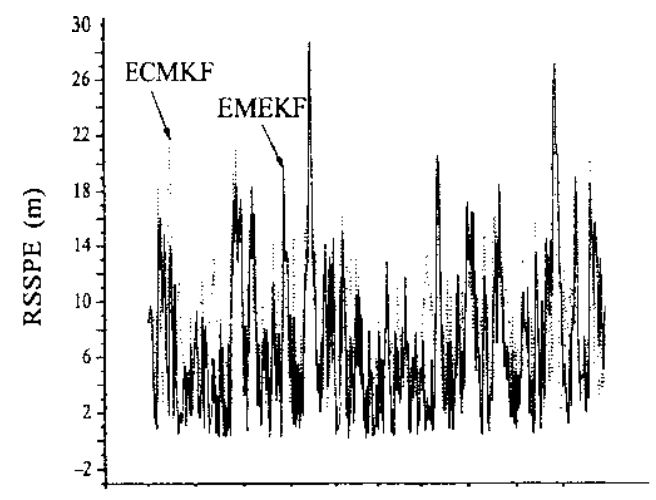

- The estimated measurement noise covariance $\mathrm{R}$ (for ECMKF) is, on an average (Fig. 6), comparable with that of CMKF-D-T and CMKF$D-M$ methods. The window length used for $R$ (ECMKF) is 10 .

- EMEKF has overall better performance in terms of percentage fit errors.

- The perforinance of CMKF-D-T. CMKF-D$\mathrm{M}, \mathrm{ECMKF}$, and EMEKF algorithms in term of percentage of fit error is compared (Fig. 7). It is clear that most of the time, EMEKF shows better performance compared to other algorithms for various sets of data 


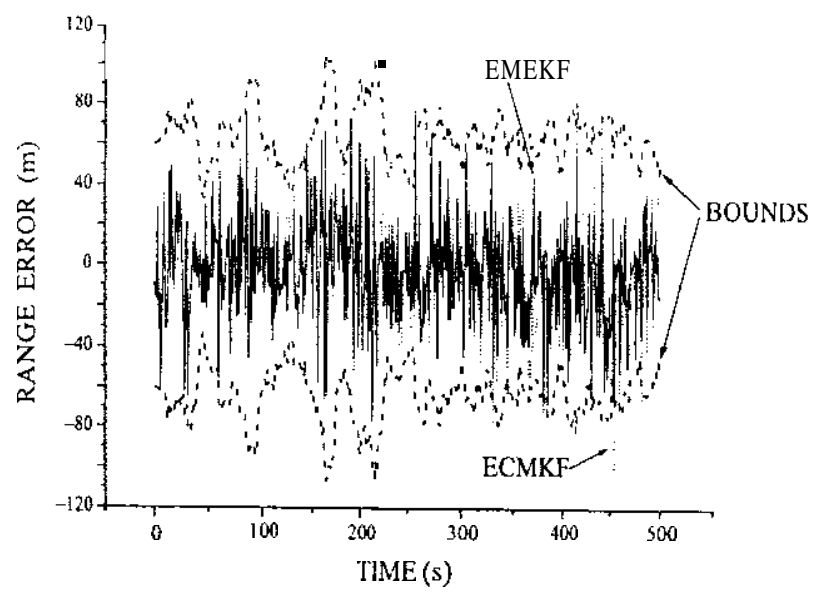

Figure 4(a). Range error comparison for EMFKF and ECMKF - simulated Set 2 data.

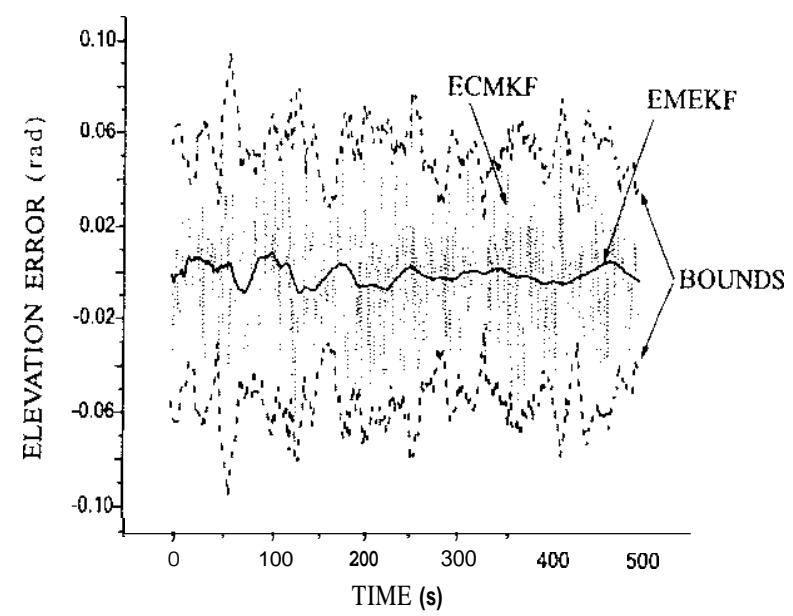

Figure 4(c). Elevation error comparison for EMEKF and FCMKF - simulated Set 2 data.

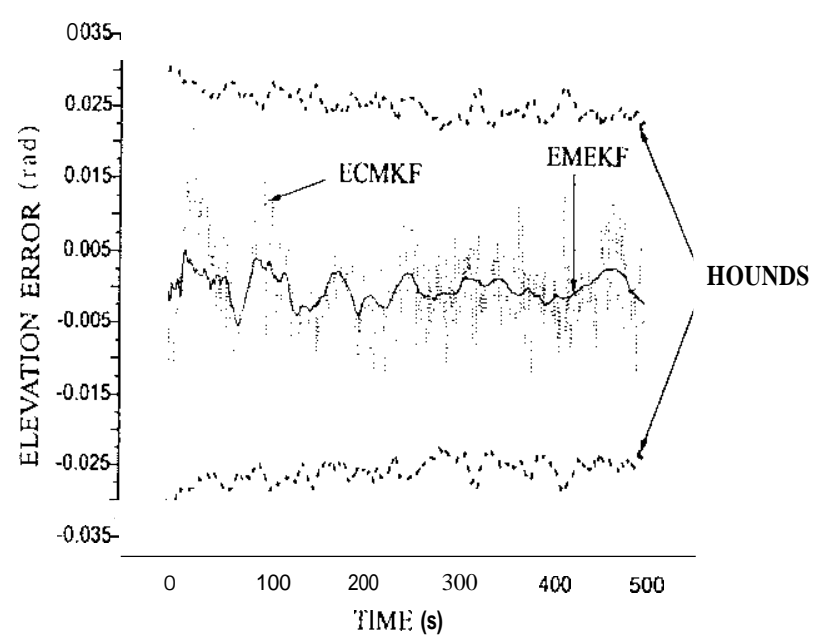

Figure 4(e). Elevation error comparison for EIEKF and ECNKF - simulated Set 2 data (Monte-Carlo simulation of 25 runs).

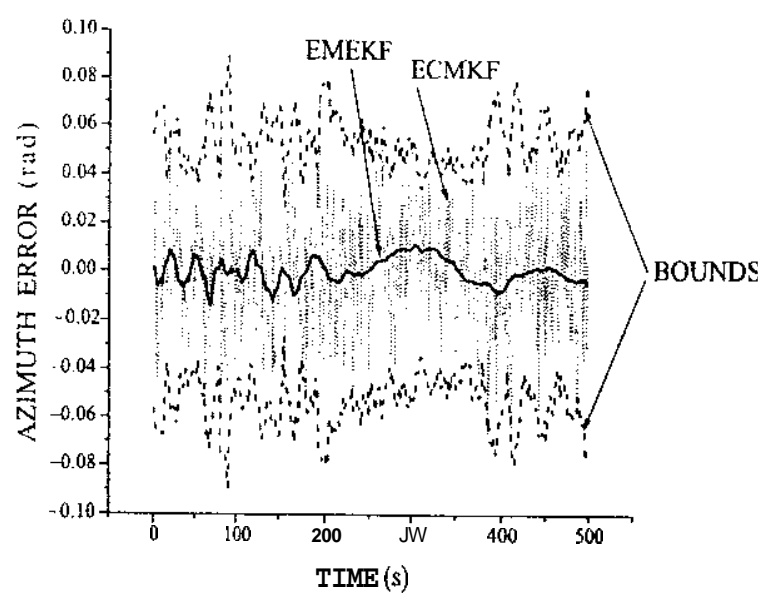

Figure 4(b). Azimuth error comparison fur EN1EKF and ECMKF - simulated Set 2 data.

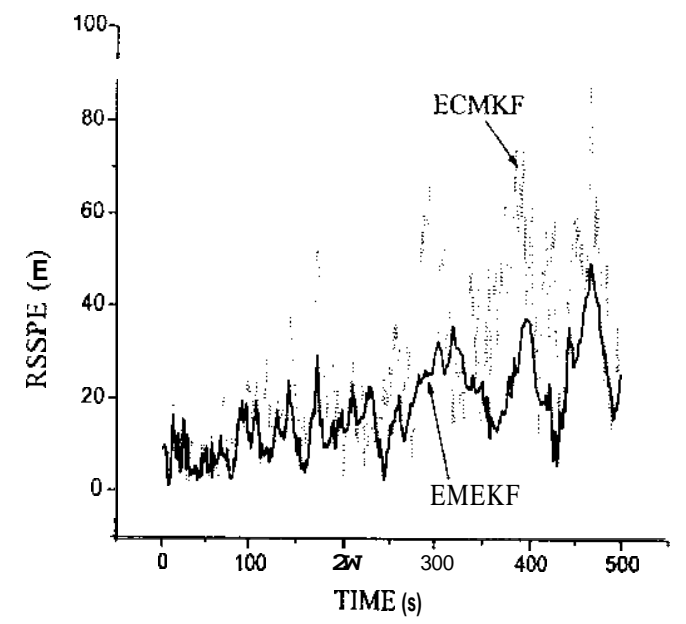

Figure 4(d). RSSPE comparison fur EMEKF and ECMKF simulated Set 2 data.

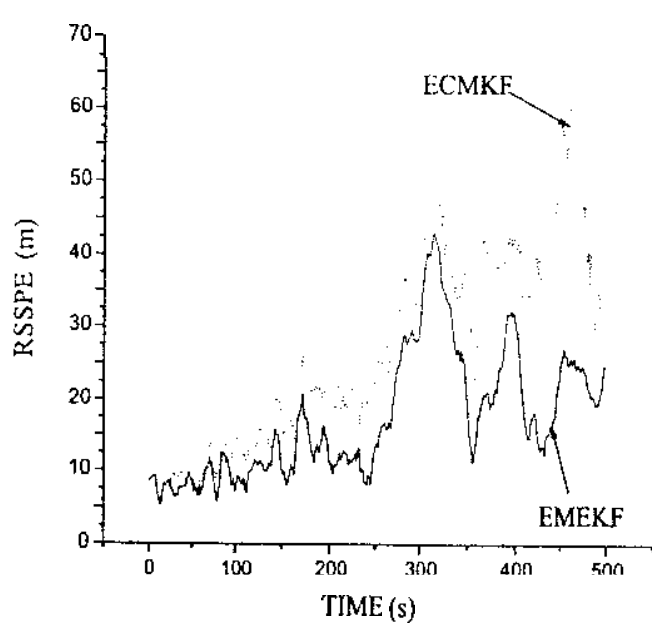

Figure 4(f). RSSPE comparison for FMIEKF and ECMJKF simulated Set 2 data (Monte-Carlo simulation of 25 runs). 


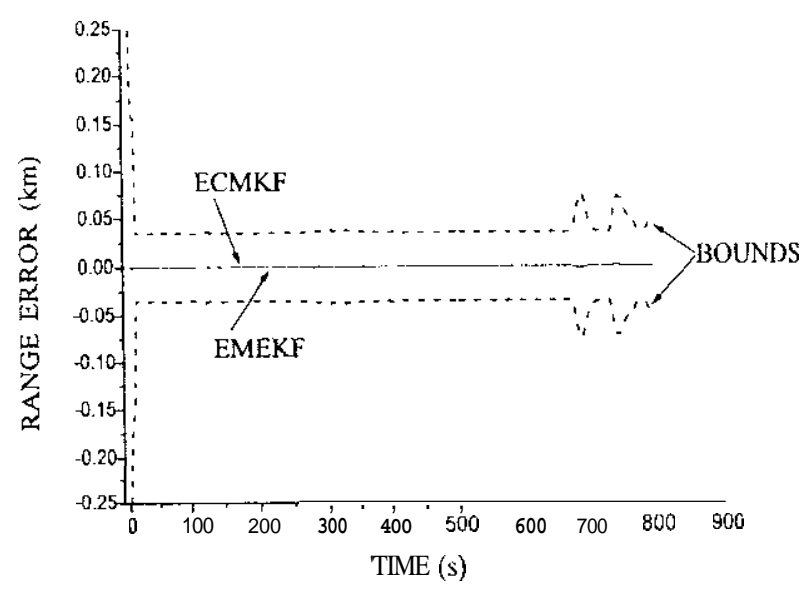

Figure 5(a). Range error comparison for ENEKF and E,CNKF - real Set 3 data.

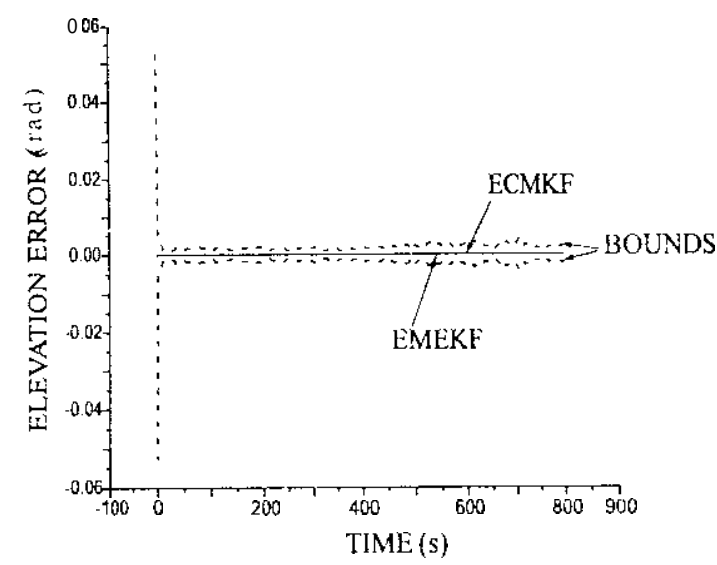

Figure 5(c). Elevation error comparison lor EMIEKF and ECMKF - real data Set 3.

\subsection{Comparison of Block and Sequential Processing of EMEKF Algorithm}

The simulated data is generated with the initial conditions: $\mid 100-100100](m)$ for position and $\left[\begin{array}{lll}5 & -5 & 5\end{array}\right](\mathrm{m} / \mathrm{s})$ for velocity, $Q=0.25 .500$ data points with a sampling interval of 1.0 s are used. Random noise was added to the true data with the following standard deviations:

$$
\sigma_{,}=3 m ; \sigma_{0}=10^{\prime \prime} ; \sigma_{\phi}=10^{\circ}
$$

A Monte-Carlo simulation of 25 runs was catried out for this data set. The sect nuinber for the process noise was kept constant. however, it was varied for the measurement noise, lor 25 runs. Fin. 8 shows the comparison of block and sequential processing techniques in term of root sum square

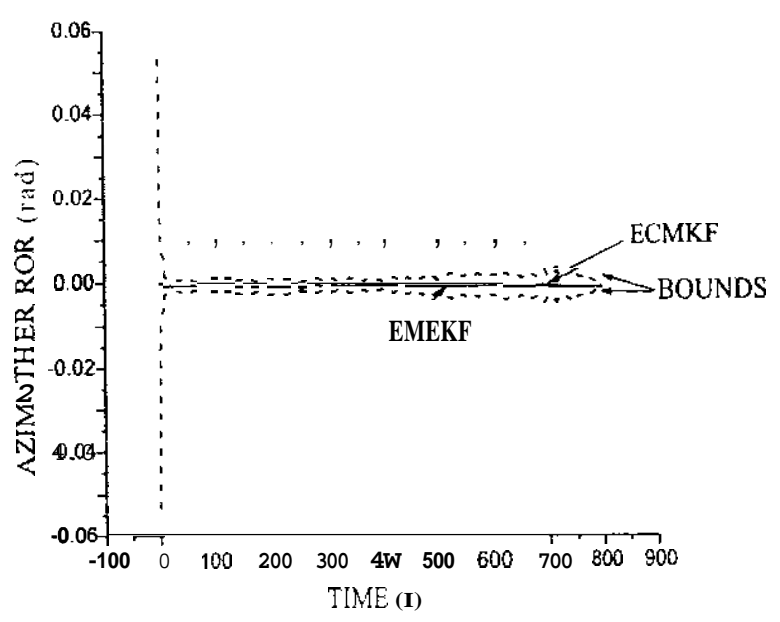

Figure 5(b). Azimuth errur comparison fur EMEKF and ECMKF - real Set 3 data.

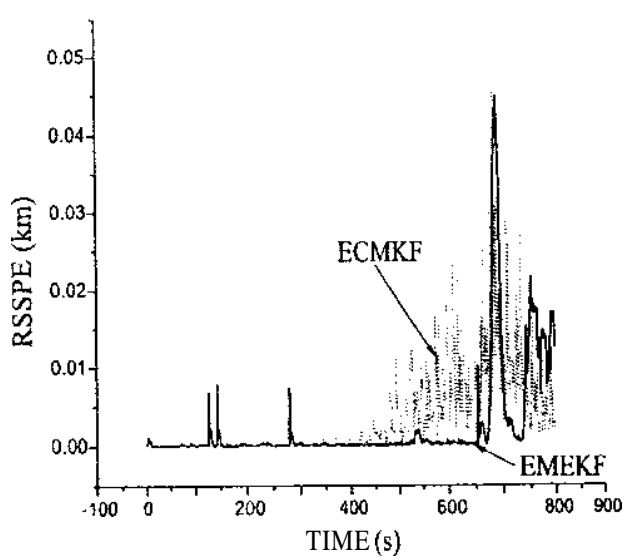

Figure 5(d). HSSPE comparison for EMEKF and ECMKFreal data Set 3 .

position error. In block processing, all the measurement arc processed at a time to update the target states (vector processing). In sequential processing the

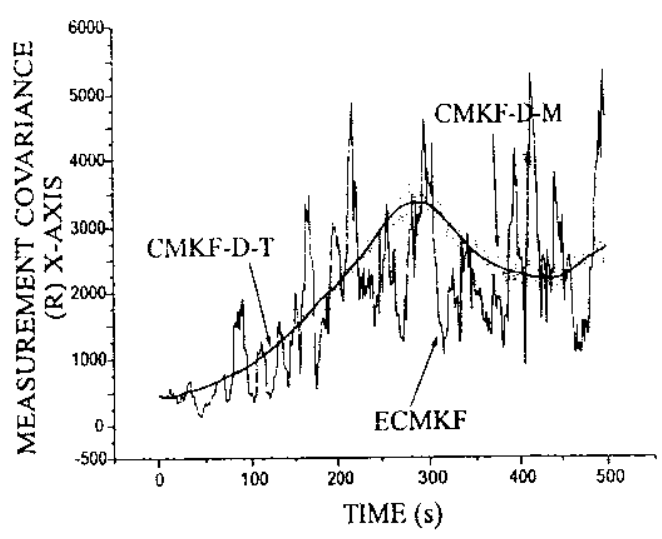

Figure 6. Measurement noise covariance estimation simulated Set 4 data. 
Table 2. Results of Set 1 data (single run)

\begin{tabular}{|c|c|c|c|c|c|c|}
\hline \multirow[t]{2}{*}{ Method } & \multicolumn{3}{|c|}{$\begin{array}{l}\text { Per cent fit error in polar frame } \\
\text { (wrt reference) }\end{array}$} & \multicolumn{3}{|c|}{$\begin{array}{c}\text { Per cent fit error in Cartesian Iranie } \\
\text { (wrt reference) }\end{array}$} \\
\hline & Range & .Azimuth & Elevation & X-pos & Y-pos & Z-pos \\
\hline ECMKF & 0.2037 & 0.3687 & 0.4160 & 02366 & 0.2465 & $0.233 \mathrm{I}$ \\
\hline EMEKF & 0.2212 & 0.0078 & 0.0085 & 0.2138 & 0.2185 & 02259 \\
\hline Method & \multicolumn{3}{|c|}{$\begin{array}{l}\text { Percent fit errur in polar frame } \\
\text { (wrt reference) }\end{array}$} & \multicolumn{3}{|c|}{$\begin{array}{c}\text { Percent fit error in Cartesian Irame } \\
\text { (wrt refcrencc) }\end{array}$} \\
\hline & Range & Azimuth & Elevation & $X$-pos & $Y_{-p o s}$ & Z-pos \\
\hline ECMKF & $0.3878(0.2993)$ & $0.9742(0.6491)$ & $0.7774(05444)$ & $1.3434(0.8935)$ & $0.7998(0.5751)$ & $0.5506(0.3065)$ \\
\hline EMEKF & $0.2393(0.1821)$ & $0.5235(0.5810)$ & $0.4806(0.2366)$ & $0.7280(0.7195)$ & $0.5625(0.4647)$ & $0.3687(0.1118)$ \\
\hline
\end{tabular}

(.) Computed based on Monte-Carlo simulation of 25 runs.

Table 4. Results of Set 3 data

\begin{tabular}{ccccccc}
\hline Method & \multicolumn{2}{c}{$\begin{array}{c}\text { Percent fit errur in polar frame } \\
\text { (wrt reference) }\end{array}$} & & Percent fit error in Cartesian frame \\
(wrt reference)
\end{tabular}

target states are updated based on the sequential processing, of measurements (ie, one measurement at a timc). In the present case. the processing order of measurement is elevation, azimuth. and range. It is clear that sequential processing in EMEKF algorithm obtains slightly better performance than the block processing. If very accurate reference data or measurements from independent sources are not available, then, the performance of the schemes is likely to degrade.

\section{CONCLUSION}

The performance of the ECMKF and EMFKF algorithms, when an accurate reference signal (say GPS) is available for getting estimate of the measurement

Table 5. Performance results of EMFKF - Set 2 data (single run)

\begin{tabular}{|c|c|c|c|c|c|c|}
\hline \multirow[t]{2}{*}{$\begin{array}{l}\text { Sequencing } \\
\text { order }\end{array}$} & \multicolumn{3}{|c|}{$\begin{array}{c}\text { Per cent fit crror in polar frame } \\
\text { (wrt reference) }\end{array}$} & \multicolumn{3}{|c|}{$\begin{array}{c}\text { Per cent fit error in Cartesian frame } \\
\text { (wrt reference) }\end{array}$} \\
\hline & Ranget & Azimath & Elevation & $X-p o s$ & Y'pos & Z-pos \\
\hline$\phi, 0, \Gamma$ & 0239 & 05235 & 04806 & 0728 & 0.562 & 0.3687 \\
\hline$\theta, \Gamma, \phi$ & 0.239 & 05234 & 0.4806 & 0727 & 0562 & 0.3686 \\
\hline I. $\phi .0$ & 0.239 & 05235 & 0.4805 & 0.727 & 0.562 & 0.3686 \\
\hline
\end{tabular}

'Sable 6. Purformance results of CMKF-D, ECMKF, and EMEKF - Set 4 data (single run)

\begin{tabular}{|c|c|c|c|c|}
\hline \multirow[t]{2}{*}{ Method } & \multicolumn{2}{|c|}{$\begin{array}{c}\text { Per rent lit error in polar Irame } \\
\text { (wrt relerence) }\end{array}$} & \multicolumn{2}{|c|}{$\begin{array}{l}\text { Per cent fit error in Cartesian frame } \\
\text { (wrt reference) }\end{array}$} \\
\hline & Range & Azimuth & X-pos & Y-pos \\
\hline CMKL-D-T & (I. 5205 & 1.4889 & 0.8432 & 1.2734 \\
\hline $\mathrm{CMKF}-\mathrm{D}-\mathrm{M}$ & 05267 & 1.4960 & 0.8404 & 1.2764 \\
\hline ECMKF & 01867 & $1.227 \mathrm{I}$ & 0.7741 & $1.158 \%$ \\
\hline FMEKF & 0.4781 & 0.6715 & 0.4062 & 05733 \\
\hline
\end{tabular}



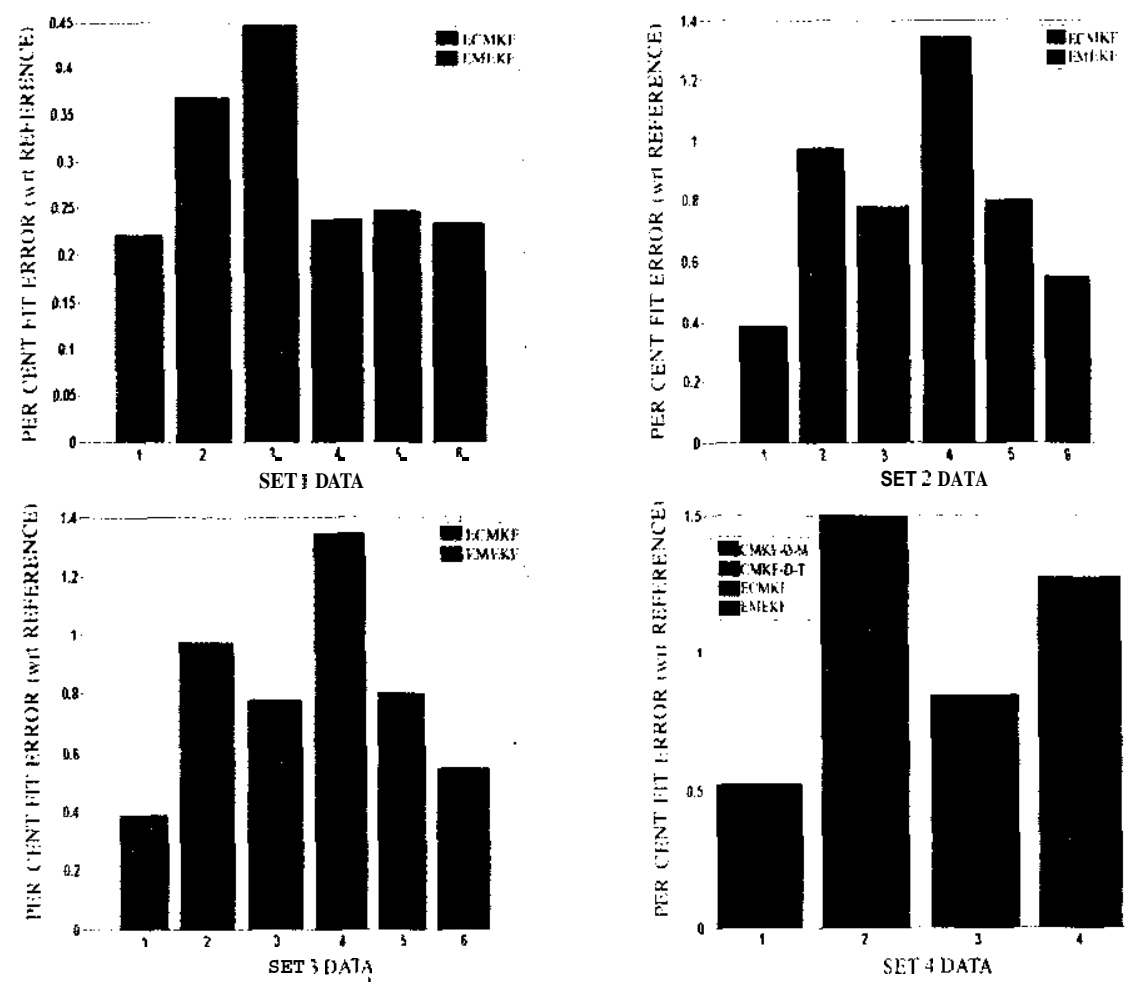

Figure 7. Performance chart to compare CMKF-D-T, CMKF-D-M, ECRIKF, and EMEKF' algorithms.

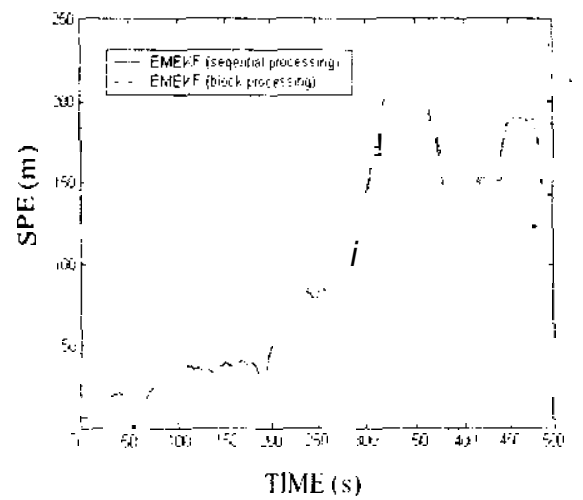

Figure 8, RSSPE comparison of sequential and block processing of EMEKF algorithm.

noise covariance. is evaluated. The algorithms are validated with three sets of data. Also the other versions of CMKF are evaluated. It has been found that the EMEKF shows better performance when the angular accuracies are low. Further. errnr modelbased CMKF gives good results.

\section{ACKNOWLEDGEMENTS}

The authors are grateful to the team, $\mathbf{A D}$ programme CCRS, DRDL. Hyderabad. for technical discussions.

\section{REFERENCES}

1. Girija, G. \& Raol. J.R. Comparison of methods for association and fusion of multisensor data for tracking applications. AIAA Guidance. Navigation, and Control Conference and Exhibit. Montreal, Quebec, Canada. Paper No. AIAA2001-4107. 6-9 August. 2001.

2. Don, Lerro \& Yaakov. Bar-Shalom. Tracking with debiased consistent converted measurements versus EKF. IEEE Trans. Aerospace Electro. Sist., 1993. 29 (3).

3. Seong-Taek. Park \& Janp. Gyu Lee. Improved Kalman filter design for three-dimension radar tracking. IEEE Trans. Aerospace Electro. Syst., 2001, 37, (2).

4. Blackman, S. Multiple target tracking with radar applications. Dedham, MA. Artech House. 1986.

5. Maybeck, Peter $\mathbf{S}$. Stochastic models, estimation and control. Vol 1. Academic Press. New York, 1979. 
6. Kashyap. Sudesh Kumar: Girija, G. \& Raol. J.K. Evaluation of converted measurement; and modified extended-Kalman filters for target tracking.
AlAA Guidance. Navigation and Control. Conference \& Exhibition. Texas, US, August $11-17,2003$.

\section{Contributors}

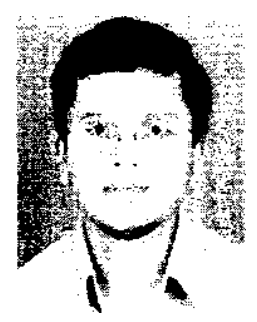

Sudesh K. Kashgap obtained his ME ! Elec Engg) from the M.S. University. Baroda. He has been working at the National Aerospace Laboratories, Bangalore. as Scientist since 2002 in the area of multisensor data fusion for target tracking. His areas of research are: Automatic Control and robotics.

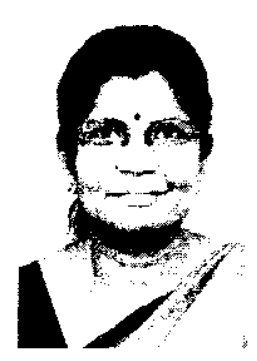

Dr (Mrs) G. Girija received her PhD from the Bangalore University in 1996. She is presently working as Scientist at the National .Aerospace Laboratories (NAL). Bangalore. Her areas of research are: Modelling, parameter estimation of aerospace vehicles and multisensor data fusion. She is a recipient of NAL Foundation Dar Award for research (1995). She has published about 50 research papers. She is a member of the Aeronautical Society of india.

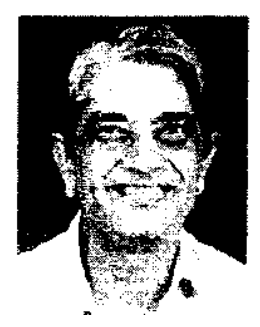

Dr J.R. Raol obtained his ME from the M.S. Eniversity, Baroda, in 1974 and PhD from McMaster University. Canada, in 1986. He worked in the National Aerospaco Laboratories, Bangalore. from 1975-8 I and was actively involved in the Multidisciplinary Control Croup's activities on human pilot modelling in fix-and-motion-based researcts simulators. He rejoined NAL in 1986 and is currently the Head of the Hight Mechanics and Control Division of NAL. His current activities are: Modelling. identification. multisensor data fusion. fumy systems, genetic algorithms and neural networks. He is a senior member of the IEEE (USA), a fellow of IEE (UK). a life fellow of the Aeronautical Society of India. and a life member of the System Society of India. He has authored (Jointly with Dr G. Girija and Dr Jatinder Singh ) a book on Modelling and Parameter Estimation of Dynamic System. He has 100 publications 10 his credit. 
The accuracy of the converted measurements depends on: (i) the geoinctry (range and bearing), and ii) on the original nieasurements. In case of large cross-range error (ic, range multiplied by bearing error), the converted measurements can have inherent bias that needs to be corrected by debiasing technique known as CMKF-D'. This technique is based on the following a priori information:

- Measurement noise inaccuracies $\sigma_{i}, \sigma_{\theta}$

- Ifthc reference data in polar frame is available, evaluation of bias and measurement noise covariance, $\mathrm{R}$, can bc done with a technique named as CMKF-D-T ( $\mathrm{T}$ is the true data).

- In case the reference data is not available, the measurement data in polar frame can be used for the same with a technique named as CMKF-D-M ( $M$ is the measured data).

- Equations used in CMKF-D-T arc reproduced- here for the sake of clarity and completeness as follows:

\section{Bias Estimation}

$$
\left[\begin{array}{l}
\mu_{x} \\
\mu_{y}
\end{array}\right]=\left[\begin{array}{l}
r \cos \theta\left(e^{-\sigma_{v}^{2} / 2}-1\right) \\
r \sin \theta\left(e^{-\sigma_{u}^{2} / 2}-1\right)
\end{array} \mid\right.
$$

where, 1 , $\theta$ are true range and bearing (ie. azimuth), whcrcas $\mu_{x}, \mu_{\text {, }}$ are bias estimated in $x$ and $y$ axes, respectively.

\section{Measurement Noise Covariance Estimation}

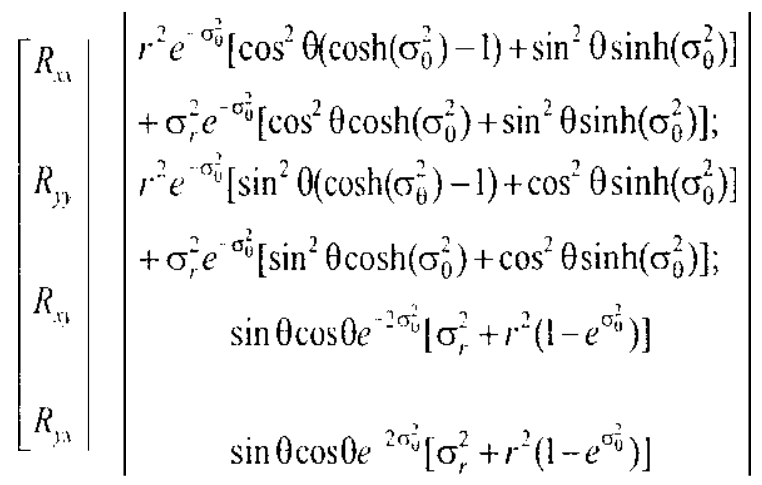

where, $R, \mathrm{~K}$ are the estimated measurement noise covariance for $x$ and $y$ axes, respectively, whereas $R$. $R_{1}$, are the measurement noise covariance across $\mathrm{x}$ and $\mathrm{y}$ axes. 
Equations used in CMKF-D-M are reproduced- for the sake of clarity and completeness as follows:

Bias Estimation

$$
\left[\begin{array}{l}
\mu_{x} \\
\mu_{y}
\end{array}\right]=\left[\begin{array}{l}
r_{m} \cos \theta_{m}\left(e^{-\sigma_{\hat{v}}^{2}}-e^{-\sigma_{\hat{u}}^{2} / 2}\right) \\
r_{m} \sin \theta_{m}\left(e^{-\sigma_{u}^{2}}-e^{-\sigma_{u}^{2} / 2}\right)
\end{array}\right]
$$

where, $r_{m}, \theta_{m}$ are measured range and bearing (ie, azimuth), whereas $\mu_{m^{\prime}} \mu_{n}$ are the bias estimated in $x$ and $y$ axes, respectively.

\section{Measurement Noise Covariance Estimation}

$$
\left[\begin{array}{l}
R_{x x} \\
R_{y y} \\
R_{x y} \\
R_{y x}
\end{array}\right]=\left[\begin{array}{l}
r_{m}^{2} e^{-2 \sigma_{\theta}^{2}}\left[\cos ^{2} \theta_{m}\left(\cosh \left(2 \sigma_{0}^{2}\right)-\cosh \left(\sigma_{\theta}^{2}\right)\right)\right. \\
\left.+\sin ^{2} \theta_{m}\left(\sinh \left(2 \sigma_{\theta}^{2}\right)-\sinh \left(\sigma_{\theta}^{2}\right)\right)\right] \\
+\sigma_{r}^{2} e^{-\sigma_{0}^{2}}\left[\cos ^{2} \theta_{m}\left(2 \cosh \left(2 \sigma_{\theta}^{2}\right)-\cosh \left(\sigma_{\theta}^{2}\right)\right)\right. \\
\left.+\sin ^{2} \theta_{m}\left(2 \sinh \left(2 \sigma_{\theta}^{2}\right)-\sinh \left(\sigma_{\theta}^{2}\right)\right)\right] \\
r_{m}^{2} e^{-2 \sigma_{0}^{2}}\left[\sin ^{2} \theta_{m}\left(\cosh \left(2 \sigma_{\theta}^{2}\right)-\cosh \left(\sigma_{\theta}^{2}\right)\right)\right. \\
\left.+\cos ^{2} \theta_{m}\left(\sinh \left(2 \sigma_{\theta}^{2}\right)-\sinh \left(\sigma_{\theta}^{2}\right)\right)\right] \\
+\sigma_{r}^{2} e^{-\sigma_{0}^{2}}\left[\sin ^{2} \theta_{m}\left(2 \cosh \left(2 \sigma_{\theta}^{2}\right)-\cosh \left(\sigma_{\theta}^{2}\right)\right)\right. \\
\left.+\cos ^{2} \theta_{m}\left(2 \sinh \left(2 \sigma_{\theta}^{2}\right)-\sinh \left(\sigma_{\theta}^{2}\right)\right)\right] ; \\
\sin \theta_{m} \cos \theta_{m} e^{-4 \sigma_{\theta}^{2}}\left[\sigma_{r}^{2}+\left(r_{m}^{2}+\sigma_{r}^{2}\right)\left(1-e^{\sigma_{0}^{2}}\right)\right] \\
\sin \theta_{m} \cos \theta_{m} e^{-4 \sigma_{0}^{2}}\left[\sigma_{r}^{2}+\left(r_{m}^{2}+\sigma_{r}^{2}\right)\left(1-e^{\sigma_{\theta}^{2}}\right)\right]
\end{array} \mid\right.
$$

The comparison of the ECMKF and CMKF-D schemes is shown in Table I and in the following figure

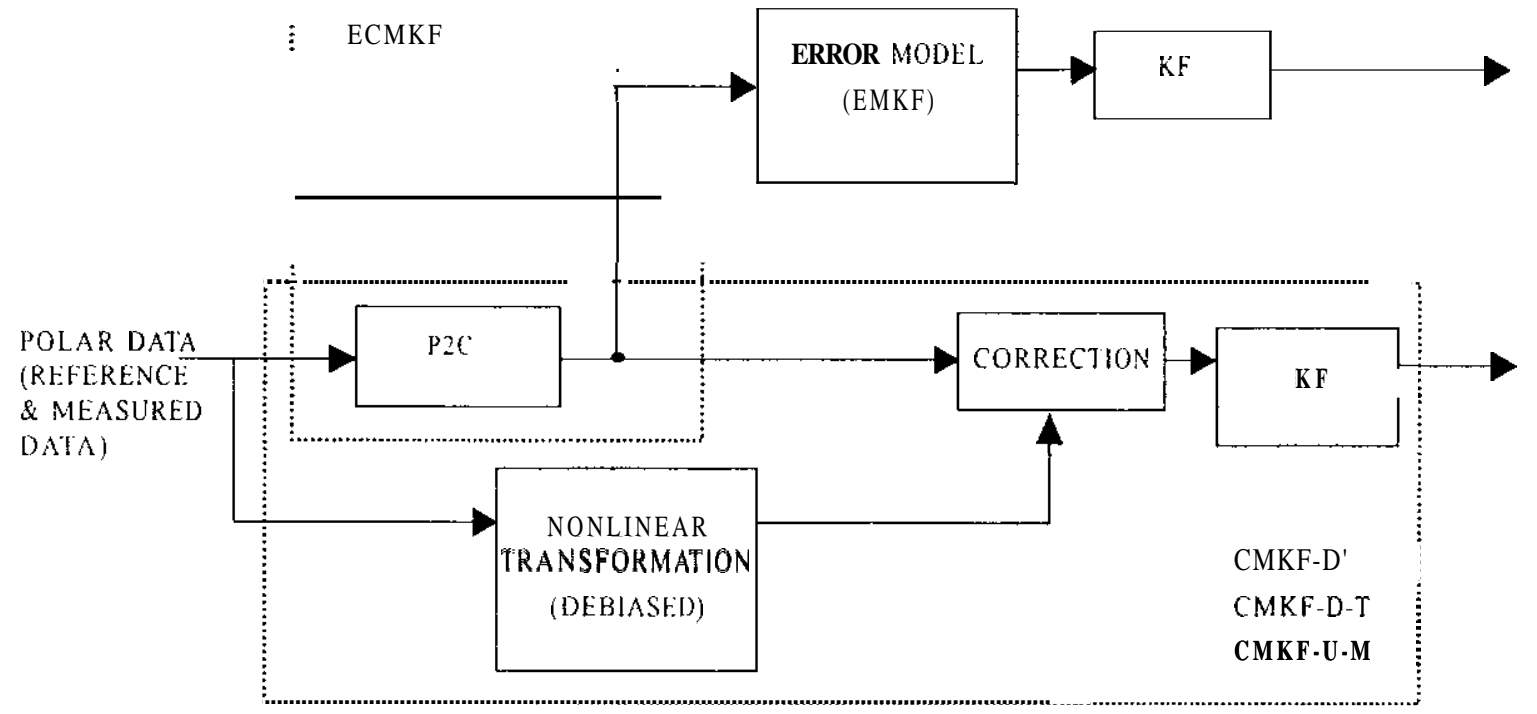

Schematic of ECMKF and CMKF-D*: T is the true reference data and $M$ is the measured data. 